\title{
Modified deep learning ResNeXt model for Human long bone fracture detection and classification
}

DOI : 10.36909/jer.ICCEMME.15777

\author{
Ashish Sharma ${ }^{1 *}$, D. P. Yadav ${ }^{2}$ \\ ${ }^{1,2}$ Department of computer Engineering \& Applications, GLA University, NH\#2, Delhi \\ Mathura Highway, Post Ajhai, Mathura (UP) India \\ *Email: ashishs.sharma@gla.ac.in; Corresponding Author
}

\begin{abstract}
The field of medical science is going to take advantage of Machine learning. It has increased dramatically over the last decade. Nowadays, you can see other innovations used in medical sciences, such as machine learning and deep learning. They can help to diagnose the illness or cause. It can also aid in the healing process by keeping notes. At a similar pace, an upper hand has been provided to the physicians for image processing by incorporating computers. Bone fractures are normal these days, and the identification of fractures is a critical part of orthopedic X-ray imaging. The automated technique lets the doctor quickly begin medical treatment. Using Machine Learning and CNN (Convolutional Neural Network), we suggest a new deep learning model perform bone diagnosis by eliminating discontinuity followed by segmentation of the image in a system that detects bone fractures. It overcomes the shortcomings of the previous approach that operates only on examination of the texture features. The proposed deep learning modified ResNeXt model performs much better than the state-of arts.
\end{abstract}

Keywords: Fracture Detection, Machine Learning, Classification, Medical Imaging, Deep Learning. 


\section{INTRODUCTION}

The demand for radiology services is going to increase day by day, the radiology services are required for the identification of the actual position of the patient. This is also needed when one has to know whether there is any fractured or not. The treatment of the bone will be effective after a proper diagnosis. The manual process is not reliable and manageable. Therefore, it is clear that changes are desperately needed in monitoring the reliability and management of the process to prevent patient. In this regard, there is a demand to design an automated system. The applications of $\mathrm{AI}$ and Machine Learning in bone images for fracture detection are growing rapidly. To solve such a problem Various ML algorithms have been technologically advanced and executed. Nowadays the researchers are working on deep learning to get better results. Dhirai et. al. has developed the model using Convolution neural systems (CNNs) that permit image assessment with local image inputs as opposed to requiring manual element building of the images.

We experimentally realize that having more perspectives accessible for a case improves the exactness of a conclusion made by a physician. Most AI algorithms assessing fracture detection just a single image, however. We needed to prove Machine learning models would improve their precision by giving progressively radiographic perspectives accessible to each case. To get the insight in the $\mathrm{x}$-ray, this research aims to convert the manual process of examining of $\mathrm{x}$ ray images. The objective of this research is to detecting fractures from X-ray images using machine learning model. Doing it manually takes a lot of effort and is vulnerable to errors. Due to noise in the images, the detection becomes cruicial task. X-rays

are more sensitive to noise, the article is focused on many pre-processing techniques for extracting noise from X-rays.

The framework can more accurately detect fractures. To improve the efficiency of the model firstly noise from the image is eradicated. The framework will delete all small objects and unwanted objects. Orthopedic surgeons may have input-ray images. To detect the fracture the 
machine uses linked components. The machine then gives feedback on whether or not the given input image has a fracture. Depending on the fractures found in the image it will go through. This machine saves time and can assist a specialist with getting his patients treated all the more proficiently.

\section{MATERIALS AND METHODS}

The available literature offers a clear roadmap for defining the research being done in this field, as well as providing a comprehensive vision for a solution.

Dimililer Kamil has created a classification scheme in that paper. The method operates in two phases, firstly, the pre-processing which defines the size and shape of the bone fracture and the another stage is labeled using the back propagation technique.

Again Dhirai et. al. have utilize simple image processing techniques in research work to identify multiple fractures within an X-ray image. Initial, a X-ray is taken as an info and afterward prehandled to wipe out clamor, then, at that point edge identification is done to disengage the edges of the bone, then, at that point division and extraction strategies are acted in which the subsequent picture is evaluated.

The MATLAB as a basic method for loading and pre-processing of images is used by Anu, T. C., and R. Raman. They have also used GLCM (Grey Level Co-occurrence Matrix) to detect the characteristics. Same as Meta classifiers and ANN, for classification decision trees are also used.

Another article is provided by Tripathi, Ankur Mani, et al; also, to detect edges in an image, it uses Canny Edge Detection and Sobel Edge Detector. It uses SVM (Support Vector Machine) to make a distinction between the fractured bones and the un-fractured bone.

Bandyopadhyay ,et. al,and Tripathi et. al. have suggested the concept of classifying bone fractures in fractured and non-fractured images. They have evaluated the performance of their methods to calculate the efficiency of the system at the last stage in their article. Firstly, they have identified the bone strength measurement, and the second is for precise examination of the 
type of fracture under error conditions.

The researchers Hoang, Nhat-Duc, and Quoc-Lam Nguyen, and Gopalakrishnan, Kasthurirangan, et al. have also developed a programmed model to distinguish and describe the fracture and non-fracture bone. Various deep learning techniques have been endeavored to render computerized image data sets. Such knowledge collections are then used to plan and validate support vector machine with AI algorithms.

In the paper of Bhandary, Abhir, et al., the authors developed a deep CNN model for the classification of lung cancer using a CT-scan image. The study compares the performance of their model with different deep learning models like VGG16, VGG19, and Resnet50. In the paper of Reischauer, Carolin, et al., the texture feature is used for the diagnosis of the prostate cancer bone. The fourth-order and the nineteen-second order GLCM texture is used for the apparent diffusion coefficient (ADC) maps and compared with the lesion. Utilizing pre-

prepared $\mathrm{CNN}$ models is an option in contrast to preparing a $\mathrm{CNN}$ all over again. Many opensource usages of CNNs are right now promptly accessible using structures. At last, the Xception coordinates the two standards for improved execution from both the Inception and Resnet architectures by Chollet F.

\section{Detailed Methodology}

Bone fraction detection and classification are performed using the Modified ResNeXt model. The ResNeXt model developed by Xie et al., was used for ImageNet classification challenge. The ResNeXt model is heavy and computation cost is high for our binary classification task. Therefore, Filter size and kernel have been changed in modified ResNeXt model to perform bone fracture detection and classification. The cardinality of the model is 32 as in the original ResNeXt model. The numbers of parameters in the model is reduced from 23x106 to 9x106.

The following figure 1, shows the image of the fracture and non-fracture bone.

\begin{tabular}{|l|l|}
\hline Fracture Bone & Healthy (Non-fracture ) Bone \\
\hline
\end{tabular}




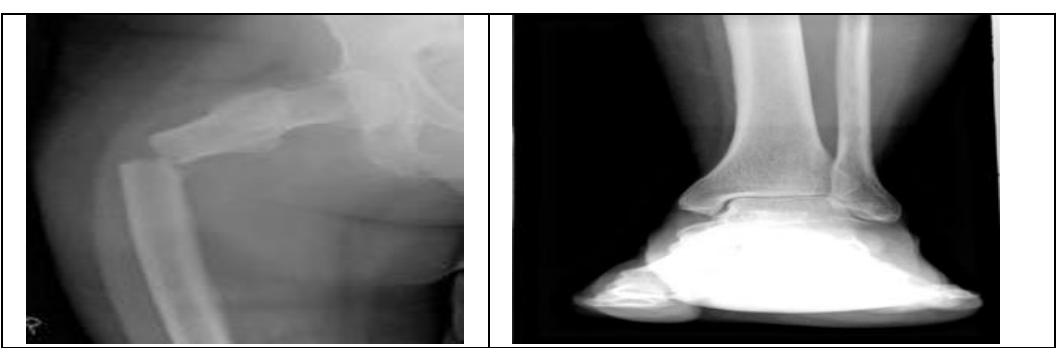

Cases for test breaks are appeared in Fig. 2 the images represents fractures in elbow, finger, forearm, hand, humerus, shoulder and wrist.

The proposed model contains residual block, Relu activation, and convolutional layers. The concept of the residual block is derived from the highway network, in which skip connection doesn't contain logical gates. This block allows the transfer of information into the last layer from the first layer of the network.

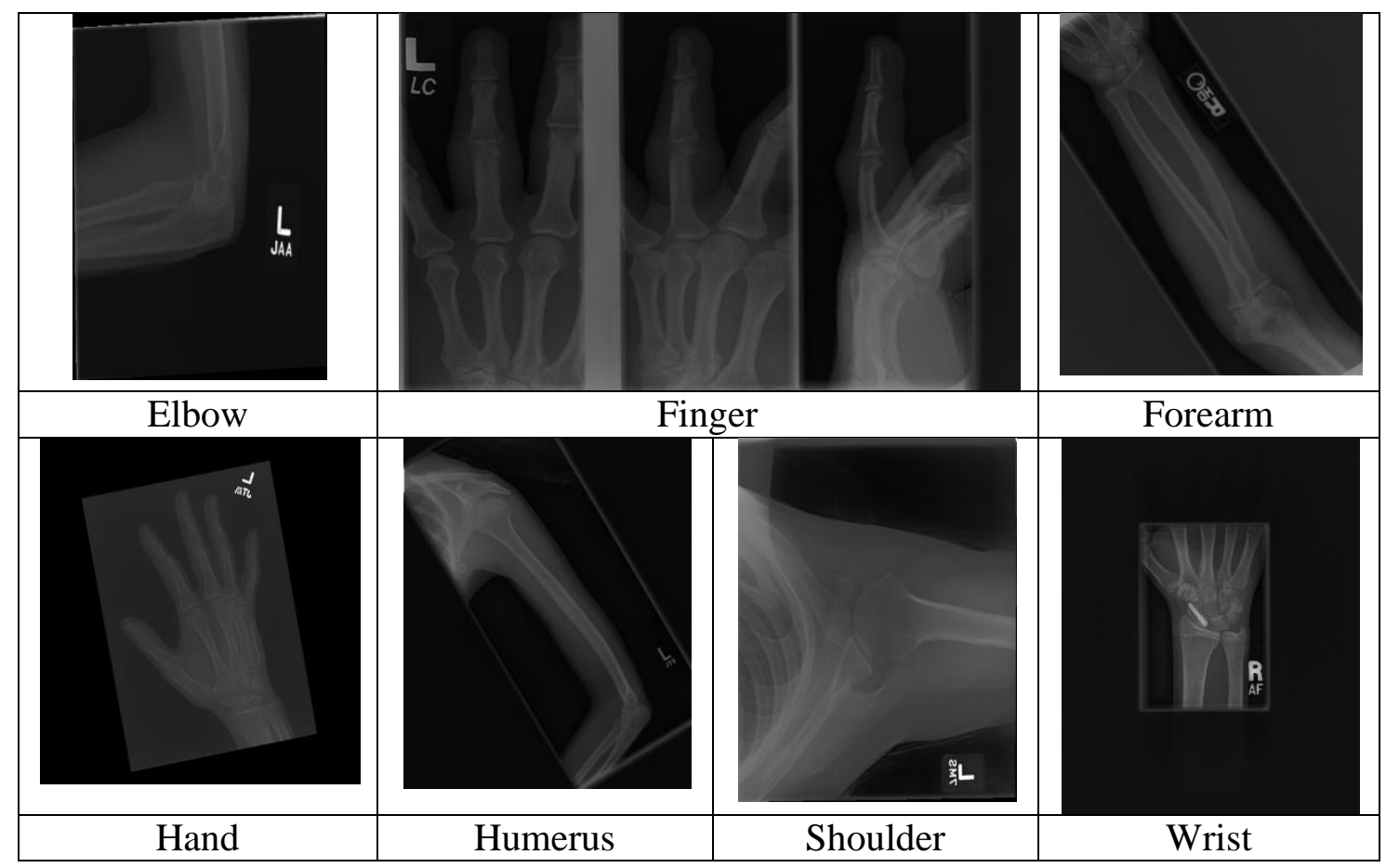


The residual blocks is defined as

$$
\alpha=\beta+\sum_{k=1}^{C} F_{k}(\beta)
$$

Where, $\alpha=$ Output of the model, $C=$ cardinality hyper-parameter,$\beta=$ input parameter vector The proposed system diagram is shown in figure 3.

The Relu activation is used to remove gradient vanishing problems. The model can learn features from the fewer number of positive input samples. The pooling layers are grouped so that the over fitting of the model can be reduced.

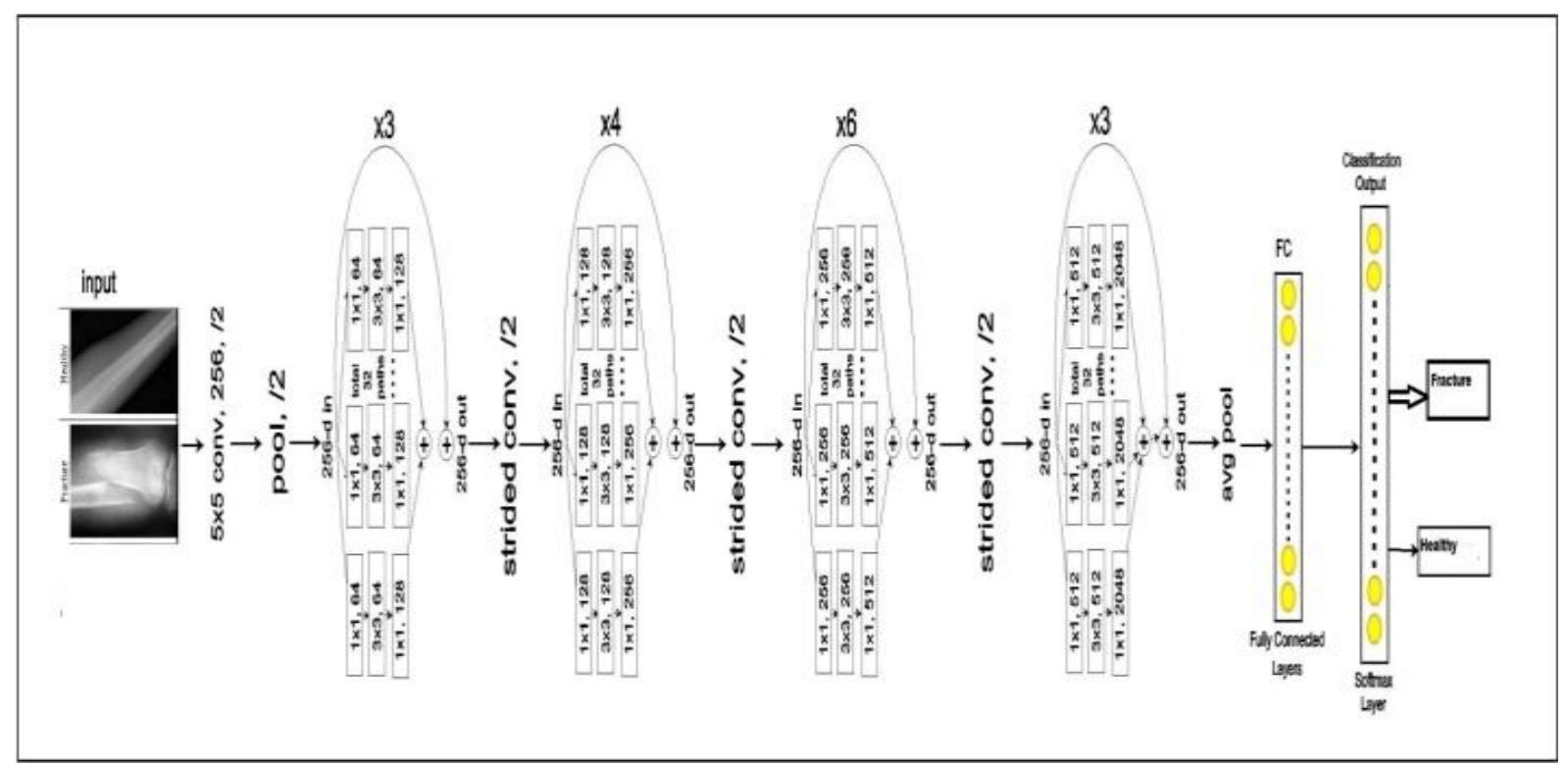

Figure 3: The proposed Machine Learning model.

The proposed model is trained with an image of $150 \times 150$ pixels. The cardinality $C=32$ maintained in the modified model. The keras 2.0 is used to implement the model and the dense layer is adjusted to 2 for this binary classification problem. This study was implemented on Nvidia GeForce GTX TITAN X GPU 


\section{RESULT ANALYSIS}

Data Set: The Diagnostic Imaging Dataset (DID) and Indian Institute of Engineering Science Technology, Shibpur (IIEST) are used in the current research.

The dataset is divided into two class fracture and non-fracture. The size of the dataset is small. In deep learning, the approaching model may get over fitted due to a small dataset. Therefore, the data augmentation technique was applied to generate new samples. The transformation techniques rotation, flipping (Horizontal and vertical), zooming, and shearing of the image are performed using image generators available in keras developed by Mikołajczyk, A., \& Grochowski, M.

After the augmentation dataset contains 34000 images. The dataset was divided into training set $80 \%$ and testing test $20 \%$. The input image of size $150 \times 150 \times 3$ is feed to the model for training and testing. The model batch size is set to 15 and trained for 20 epochs. The training and validation accuracy of the model is calculated, shown in figure 4 . The classification accuracy of the model is more than $92 \%$. The training and validation loss is less than 0.5 . The training and validation loss is shown in the figure 5 .

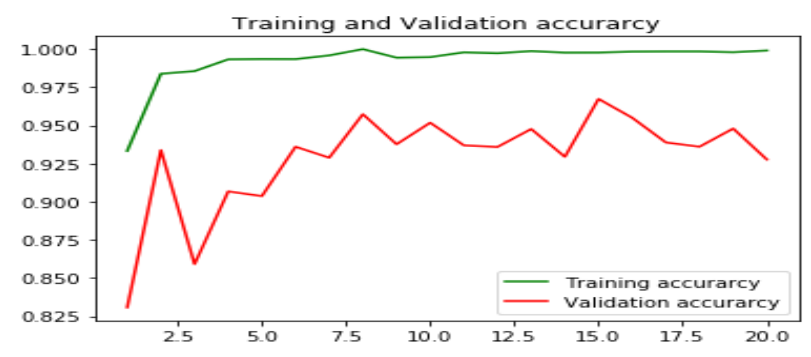

Figure 4: Training and validation accuracy

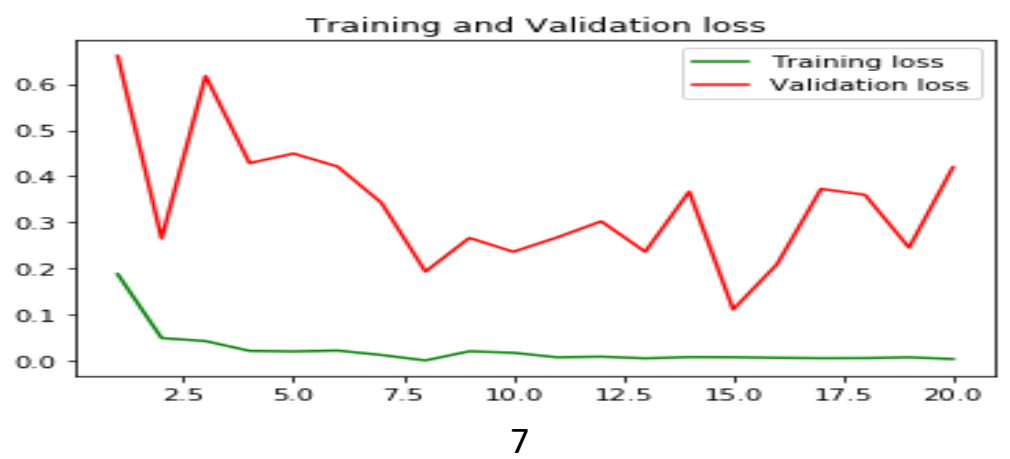


Figure 5: Training and validation loss of the model

We calculated three readings from our device and a normal medical device which made us clear about how accurate our device is, results and comparison are mentioned above

\section{DISCUSSION:}

A bone fracture is a common disease now a day. A bone fracture diagnosis is essential as soon as possible. So that proper treatment will be provided to the patient. Past researches on bone diagnosis have used machine learning techniques like SVM, ANN, random forest, etc. These types of machine learning techniques are train by feeding features extracted from the image. Due to this performance depends on the human. In a deep learning model features are automatically learned by the model from the image. The major concern in a deep learning model is the large size of the dataset for training. In the past several types of research on medical imaging have been successfully conducted using a deep learning approach.

Kitamura, G., Chung, C. Y., \& Moore, B. E. have classifies ankle bone into a fracture and nonfracture using deep learning models. Their method compares the performance of different pretrained models likes Inception V3, Resnet, Resnet with drop/aux, Xception, Xception with drop/aux. The accuracy of these models is not optimal on their dataset. The maximum accuracy achieved is $78 \%$. Therefore, they design a new deep learning model to improve the accuracy of the model. The accuracy of the model is $81 \%$. The computation time of the model is high and the model needs to train with more data.

The fracture detection model proposed by Kim, D. H., and T. MacKinnon also uses a transfer learning approach. Their method can classify the fracture and non-fractured bone using Inception V3 model. To improve the size of the dataset to 11112 images, the data augmentation technique was also applied. The 2000 epochs were used for the training of the model. The ROC curve is 0.954 , sensitivity 0.9 , and specificity 0.88 . Further, a deep learning model can also be 
designed to improve the accuracy of the model and training with more

samples.

In the proposed model data augmentation technique is applied to generate more samples and dataset size is extended to 34000 . The modified ResNeXt model is 50 layers of deep learning models. The model is trained with 20 epochs on a GPU. The final results in the model is shown in the form of the confusion matrix in the table 1.

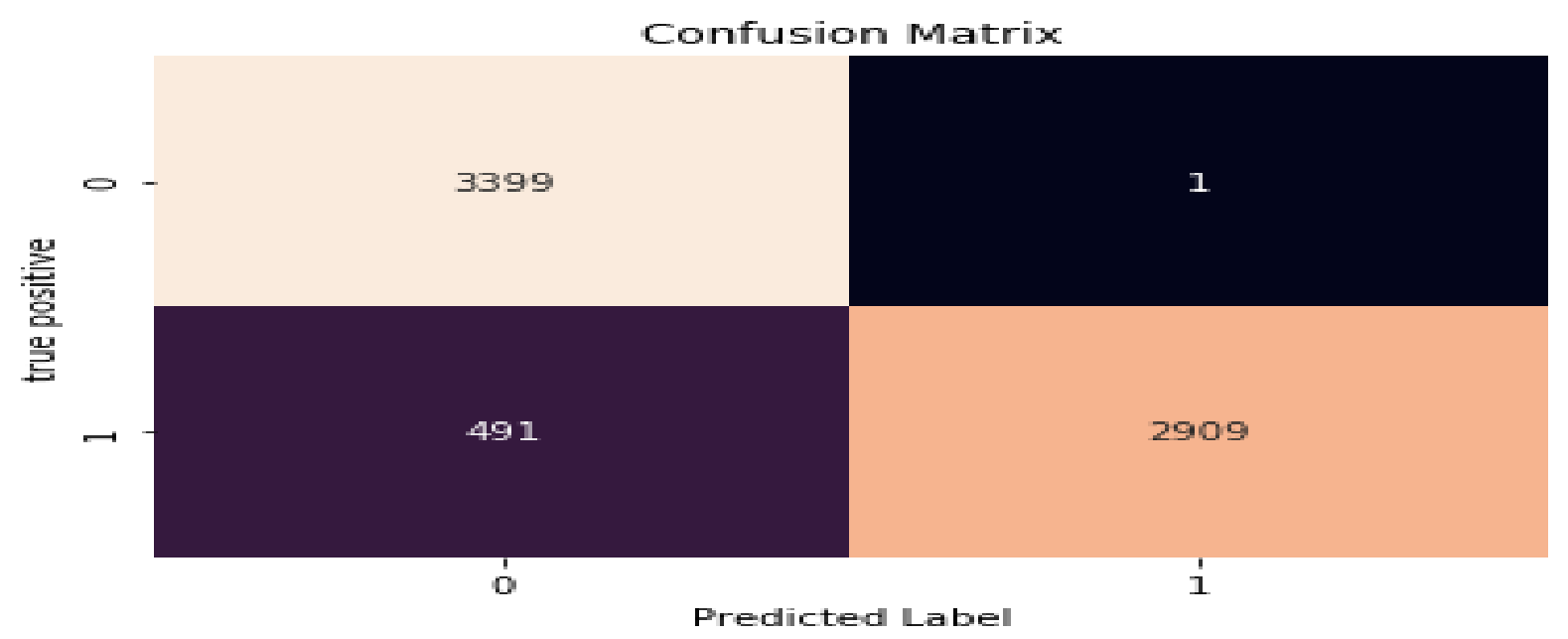

Table 1: The result of the proposed model.

The performance measures accuracy precision, recall, and f1-score are calculated from the confusion matrix to evaluated model performance. The performance measures are shown in the table 2.

Accuracy: The accuracy of the model is calculated by a total number of the test image to the total number of correctly predicted by the bone diagnosis model.

$$
\text { Accuracy }=\left(\frac{T P+T N}{T P+T N+F P+F N}\right)
$$

Precision: The precision of the model is evaluated by total number of true positive samples to the total number of correctly observation predicted by the model.

$$
\text { Precision }=\left(\frac{T P}{T P+F P}\right)
$$

Recall: The recall of the model is calculated using total number of positive sample to the total number of observations in that class. 


$$
\operatorname{Re} \text { call }=\left(\frac{T P}{T P+F N}\right)
$$

F1-score: It is the harmonic mean of the precision and recall

$$
F 1-\text { score }=2 \times\left(\frac{\text { Pr ecision } \times \text { Re call }}{\operatorname{Pr} \text { ecision }+\operatorname{Re} \text { call }}\right)
$$

\begin{tabular}{|c|c|c|c|}
\hline Types of Bone & Precision & Recall & F1-score \\
\hline Fracture & 0.87 & 1 & 0.93 \\
\hline Healthy (Non-fracture )Bone & 1 & 0.86 & 0.92 \\
\hline
\end{tabular}

Table 2: The Precision, Recall and F1-score of the proposed model.

The ROC (Receiver Operating Characteristic) curve is also plotted to measure the performance of the model by Goodfellow, I., Bengio, Y., \& Courville, A. The proposed model is able to cover more than $92 \%$ of the curve.

The recall for fracture bone is very high and F-1 score of the model for fracture and nonfracture bone is also high. The exhibition of the proposed model is far superior to the state-of arts.

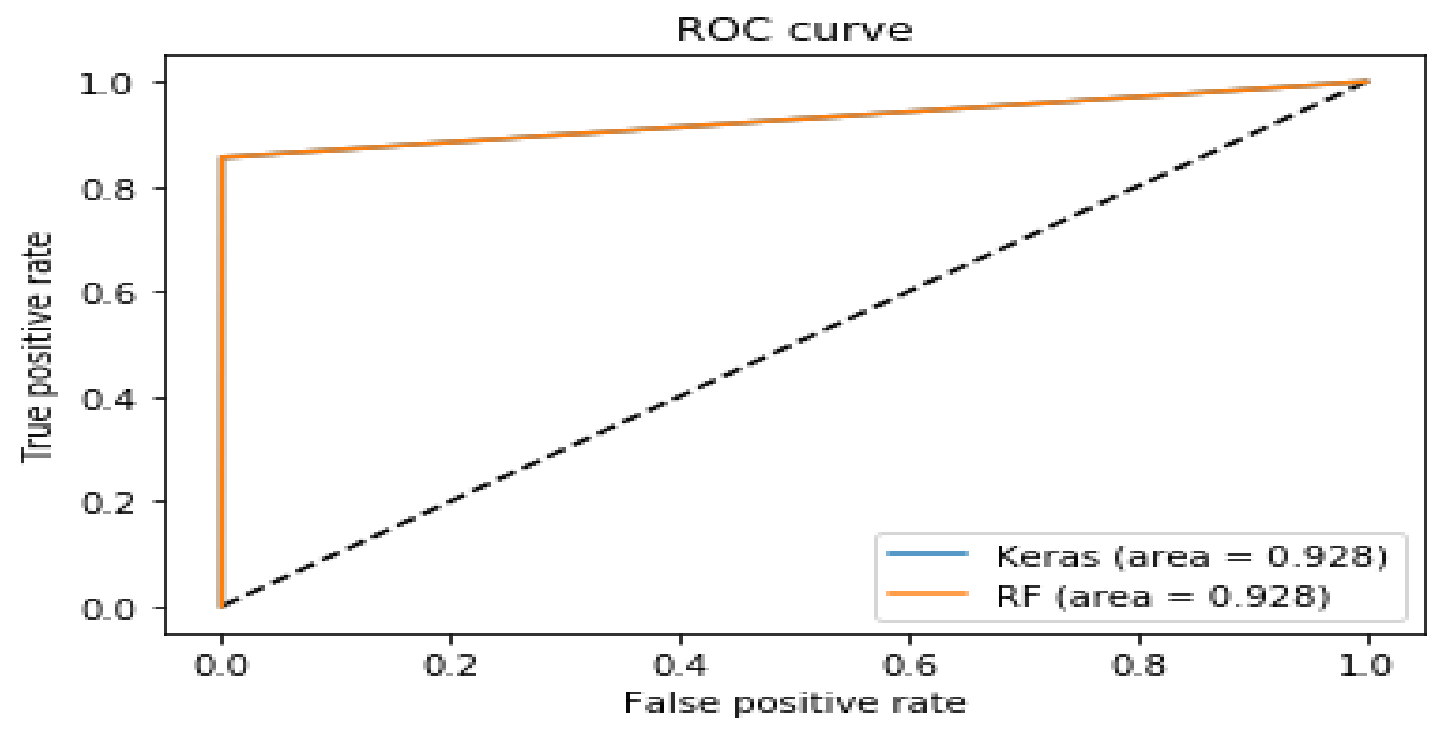

Figure

6: The ROC of the proposed model 


\section{CONCLUSION}

The bone fracture diagnosis performed using manually is time-consuming. Therefore, we have developed a deep learning-based model for the diagnosis of the bone. Deep learning requires a large image dataset for training. Therefore, we have performed a data augmentation technique. The past researches on the bone diagnosis have used less training image and less deep model. Due to this, the performance is not optimal. The modified ResNeXt model is many 50 layers deep. The overfitting of the model is taken care of by residual block and data augmentation techniques. The classification accuracy of the proposed model is acceptable. Also, the training and validation losses are less. The model performance can be further improved by more training on bone image samples. The model needs to test on the real-time dataset so that further performance can be measured. In future research we, will try to implement a deep learning model to calculate the length of the fractured part, so that surgery of the bone can be done efficiently.

\section{REFERENCES}

Abadi M, Agarwal A, Barham P, Brevdo E, Chen Z, Citro C, et al: TensorFlow: Large-Scale Machine Learning on Heterogeneous Distributed Systems [Internet]. arXiv [cs.DC]. 2016. Available from: http://arxiv.org/abs/1603.04467. Accessed 9 Feb 2018

Anu, T. C., and R. Raman. Detection of bone fracture using image processing Methods. International Journal of computer applications 975 (2015): 8887.

Bandyopadhyay, Oishila, Arindam Biswas, and Bhargab B. Bhattacharya. Long-bone fracture detection in digital X-ray images based on digital-geometric techniques. Computer methods and programs in biomedicine 123 (2016): 2-14.

Bhandary, Abhir, et al. Deep-learning framework to detect lung abnormality-A study with chest X-Ray and lung CT scan images. Pattern Recognition Letters 129 (2020): 271-278.

Chollet F: Xception: Deep Learning with Depthwise Separable Convolutions [Internet]. arXiv 
[cs.CV]. 2016. Available from: http://arxiv.org/abs/1610.02357. Accessed 9 Feb 2018

Dimililer, Kamil. IBFDS: Intelligent bone fracture detection system. Procedia computer science 120 (2017): 260-267.

Dhiraj B. Bhakare, Prajwal A. Jawalekar, Sumit D. Korde. A Novel Approach for Bone Fracture Detection using Image Processing” International Research Journal of Engineering and Technology (IRJET),2018.

Gopalakrishnan, Kasthurirangan, et al. Deep Convolutional Neural Networks with transfer learning for computer vision-based data-driven pavement distress detection. Construction and Building Materials 157 (2017): 322-330.

Goodfellow, I., Bengio, Y., \& Courville, A. (2016). Deep learning. MIT press.

Hoang, Nhat-Duc, and Quoc-Lam Nguyen. A novel method for asphalt pavement crack classification based on image processing and machine learning." Engineering with Computers 35.2 (2019): 487-498.

Jones E, Oliphant T: Peterson - URL http://scipy.org P, 2011. SciPy: Open source scientific tools for Python, 2009. 2011

Kim, D. H., and T. MacKinnon. Artificial intelligence in fracture detection: transfer learning from deep convolutional neural networks." Clinical radiology 73.5 (2018): 439-445.

Kitamura, G., Chung, C. Y., \& Moore, B. E. (2019). Ankle fracture detection utilizing a convolutional neural network ensemble implemented with a small sample, de novo training, and multiview incorporation. Journal of digital imaging, 32(4), 672-677.

Li Z, Wang C, Han M, Xue Y, Wei W, Li L-J, et al: Thoracic Disease Identification and Localization with Limited Supervision [Internet]. arXiv [cs.CV]. 2017. Available from: http://arxiv.org/ abs/1711.06373. Accessed 9 Feb 2018

Mikolajczyk, A., \&Grochowski, M. (2018). Data augmentation for improving deep learning in image classification problem. In 2018 international interdisciplinary $\mathrm{PhD}$ workshop (IIPhDW) (pp. 117-122). IEEE.

Reischauer, Carolin, et al. Texture analysis of apparent diffusion coefficient maps for treatment 
response assessment in prostate cancer bone metastases - a pilot study." European journal of radiology 101 (2018): 184-190.

Sharma, Ashish, Anant Ram, and Archit Bansal. Feature Extraction Mining for Student Performance Analysis." Proceedings of ICETIT 2019. Springer, Cham, 2020. 785-797

Tripathi, Ankur Mani, et al. Automatic detection of fracture in femur bones using image processing." 2017 International Conference on Innovations in Information, Embedded and Communication Systems (ICIIECS). IEEE, 2017.

Tripathi, A. M., Upadhyay, A., Rajput, A. S., Singh, A. P., \& Kumar, B. (2017, March). Automatic detection of fracture in femur bones using image processing. In 2017 International Conference on Innovations in Information, Embedded and Communication Systems (ICIIECS) (pp. 1-5). IEEE.

Xie, S., Girshick, R., Dollár, P., Tu, Z., \& He, K. (2017). Aggregated residual transformations for deep neural networks. In Proceedings of the IEEE conference on computer vision and pattern recognition (pp. 1492-1500).

Yadav, D. P., et al. "Feature Extraction Based Machine Learning for Human Burn Diagnosis From Burn Images." IEEE Journal of Translational Engineering in Health and Medicine 7 (2019): 1-7.

https://www.england cancerimagingarchive.net/collections/, last accessed on Dec 18, 2019. https://www.iiests.ac.in/, last accessed on Dec 18, 2019.

https://www..nhs.uk/statistics/statisticalwork-areas/diagnostic-imaging-dataset/. 